\title{
Star formation in the nuclear region of M83 The HST/NICMOS view
}

\author{
T. Wiklind ${ }^{1}$, A. Lundgren-Andersson ${ }^{2,3}$ and H. Olofsson ${ }^{2}$ \\ ${ }^{1}$ ESA Space Telescope Division, STScI, 3700 San Martin Dr, Baltimore MD 21218, USA \\ email: wiklind@stsci.edu \\ ${ }^{2}$ Stockholm Observatory, AlbaNova, SE-10691 Stockholm, Sweden \\ ${ }^{3}$ European Southern Observatory, Casilla 19001, Santiago 19, Chile
}

\begin{abstract}
The nuclear region of the barred spiral galaxy M83 has been imaged at near-infrared wavelengths with the HST NICMOS, showing a new region of star formation activity.
\end{abstract}

\section{Introduction}

Several mechanisms have been proposed to facilitate the transfer of gas inwards to galactic nuclei, and angular momentum towards the outer regions of galaxies. The aim is usually to find a source for fueling an Active Galactic Nucleus (AGN). The proposed mechanisms include gravitational galaxy-galaxy interaction, merging and the presence of a barred potential. Although several of these mechanisms have been identified in AGNs, no clear connection between AGN activity and the presence of these mechanisms have been established (see Schmitt, these proceedings). An alternative sink for gas flowing inwards is star formation. Nuclear starbursts are often found in connection with AGN activity, but can also exist without any observable AGN. In fact, the fuel requirement for sustaining a nuclear starburst is often more demanding than fueling an AGN.

The barred grand-design spiral galaxy M83 represents a nearby $(4.5 \mathrm{Mpc})$ example of system which includes at least two, perhaps three, of the mechanisms proposed for transfering gas to the central regions: it has a strongly barred potential, it is believed to have gravitationally interacted with its neighbor NGC5253 and, the presence of a double nucleus (Thatte et al. 2000) could hint at a merging dwarf galaxy. In addition, M83 has a relatively large gas content (Lundgren et al. 2004), exhibits wide-spread star formation over the optical disk, as well as a nuclear starburst (Harris et al. 2001). M83 is a late type spiral and has a small bulge. A massive black hole in the center would be of only moderate size and luminosity. Due to M83's small distance, however, even a small central black hole would be detectable if it radiates at a few percent of its Eddington luminosity. Despite detection of numerous radio and X-ray point sources in M83 (Cowan \& Branch 1985; Sori \& Wu 2002) there are no indications of any AGN activity in M83. All of the emission can be attributed to star formation.

\section{Observations and results}

We have used near-infrared NICMOS HST images to study the central region of M83 in detail. The observations were done in May 1998 using all three NICMOS cameras (proposal 7218, PI. M.J. Rieke). We retrieved NIC2 and NIC3 data from the HST archive. The pixel sizes of the NIC2 and NIC3 cameras are 0..076 and $0 . .203$, respectively, corresponding to $1.7 \mathrm{pc}$ and $4.4 \mathrm{pc}$ at the assumed distance of M83 of $4.5 \mathrm{Mpc}$. 

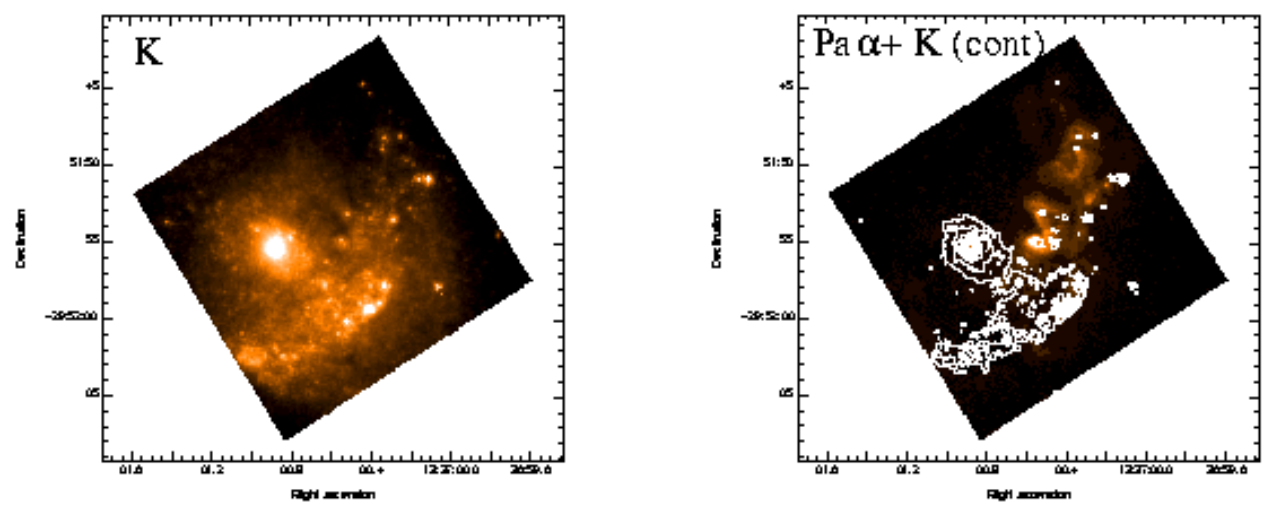

Figure 1. Left: A K-band image of the nuclear region of M83 obtained with the NICMOS camera on the HST. Right: Pa $\alpha$ emission with the K-band emission shown in contours.

Line emission from $\mathrm{H}_{2}$, Pa $\alpha$, FeII and HeI were observed in narrow band images. The CO bandhead absorption was imaged in a medium band filter. The narrow line images containing emission lines, as well as the medium band image of CO absorption, all had corresponding narrow (medium) band images only containing continuum emission. The latter were subtracted from the line containing images. A broad band image using the F160W filter corresponds closely to the standard H band, while the F222M filter corresponds to the $\mathrm{K}$ band.

The main features of the $2.2 \mu \mathrm{m}$ continuum image of M83 nuclear region were already identified in a ground-based $\mathrm{K}$ band image obtained with the 3.6m CFHT on Mauna Kea (Gallais et al. 1991). Subsequent observations, both from the ground (Elmegreen et al. 1998) and now from the HST (see also Thatte et al. 2000) show the same features, albeit in much better detail. The NICMOS narrow band line images, however, show that the nucleus is very different from the continuum images, with star formation going on in previously unknown locations.

In Fig. 1 we show the K-band image, with the infrared peak and the arc with HII regions clearly visible. These two features are also prominent in optical images (cf. Harris et al. 2001). In the right hand part of Fig. 1 we show the continuum subtracted Pa $\alpha$ image, with the K-band continuum emission overlayed as contours. The Pa $\alpha$ emission is concentrated in a region $\sim 3^{\prime \prime}$ west of the infrared peak. The Pa $\alpha$ peak could be associated with the second dynamical center identified through long slit IR spectroscopy (Thatte et al. 2000). Very little $\mathrm{Pa} \alpha$ emission is associated with the infrared peak region. The CO absorption suggests that the $\mathrm{Pa} \alpha$ emission is associated with the youngest stellar population, while the K-band emission is associated with a population of age $\sim 10^{7}$ years. Apparently, star formation has recently occured in two episodes, one which is currently on-going and one where the peak of activity occured $\sim 10$ Myrs ago. The two star formation episodes appears to be associated with the two different dynamical centers of M83.

\section{References}

Cowan, J. J., \& Branch, D. 1985, ApJ, 293, 400

Elmegreen, D. B., Chromeym F. R., \& Warren, A. R. 1998, AJ, 116, 2834

Gallais, P., Rouan, D., Lacombe, F., Tiphène, D., \& Vauglin, I. 1991, A\&A, 243, 309

Harris, J., Calzetti, D., Gallagher, J. S., Conselice, C. J., \& Smith, D. 2001, AJ, 122, 3046

Lungren, A. A., Wiklind, T., Olofsson, H., \& Rydbeck, G. 2004, A\&A, 413, 505

Soria, R., \& Wu, K. 2002, A\&A, 384, 99

Thatte, N., Tecza, M., \& Genzel, R. 2000, A\&A, 364, L47 